07

\title{
Численное и экспериментальное исследования оптимизированного p-SOS-диода
}

\author{
() А.Г. Люблинский, Е.И. Белякова, И.В. Грехов \\ Физико-технический институт им. А.Ф. Иофрфе РАН, \\ 194021 Санкт-Петербург, Россия \\ e-mail: Alexander.Lyublinsky@mail.ioffe.ru
}

(Поступило в Редакцию 25 мая 2018 г.)

\begin{abstract}
Представлены результаты экспериментального исследования и численного моделирования процесса переключения $p^{+} P_{0} n^{+}$-структуры SOS-диода с уменьшенной толщиной $P$-базы. Предложенная одномерная диффузионно-дрейфовая модель динамики электронно-дырочной плазмы дала хорошее согласие с экспериментом. Уменьшение толщины $P$-базы позволило повысить амплитуду выходного импульса в два раза при той же плотности коммутируемого тока. Это было достигнуто за счет существенного снижения коммутационных потерь, а также за счет формирования в процессе обрыва тока области сильного электрического поля квазипрямоугольной формы на $P_{0} n^{+}$-переходе. В результате были сформированы выходные импульсы напряжения с амплитудой, существенно превышающей напряжение статического пробоя $P_{0} n^{+}$-перехода $p$-SOS-диода. Этот эффект наблюдался впервые для высоковольтных полупроводниковых ключей размыкающего типа.
\end{abstract}

DOI: 10.21883/JTF.2019.03.47177.208-18

\section{Введение}

Возможность наносекундного размыкания тока с плотностью до нескольких десятков $\mathrm{kA} / \mathrm{cm}^{2}$ при переключении из прямого смещения на обратное в кремниевом диоде со структурой $p^{+} p^{\prime} N_{0} n^{+}$и сверхглубоким $(150-200 \mu \mathrm{m}) p^{\prime} N_{0}$-переходом в кремнии $n$-типа проводимости была показана в [1]. Численное моделирование этого процесса, названного авторами SOS-эффектом (SOS-Semiconductor Opening Switch), было проведено в [2-4] и др. Моделирование, в частности, показало, что процесс обрыва нарастающего импульса обратного тока начинается с образования у $n^{+} N_{0}$-границы узкой области электрического поля. Этот пик поля быстро достигает напряженности, близкой к пробивным значениям, но в дальнейшем его положение, а также амплитуда и ширина остаются практически неизменными. Затем формируется вторая область сильного поля в $p^{\prime}$-слое. Этот домен сильного поля быстро расширяется в потоке свободных дырок, выносимых из плазмы, и именно он дает основной вклад в процесс обрыва тока и роста напряжения на SOS-структуре. K моменту достижения максимума напряжения на SOS-диоде в нем образуются две области сильного поля с амплитудой до $150-200 \mathrm{kV} / \mathrm{cm}$ : широкая в $p^{\prime}$-слое и узкая у $n^{+} N_{0}$-границы, разделенные областью шириной 50-60 $\mu \mathrm{m}$, заполненной электроннодырочной плазмой с концентрацией, примерно на порядок превышающей уровень легирования. Таким образом, в момент переключения тока в параллельно подключенную нагрузку, $p^{\prime} N_{0}$-переход и бо́льшая часть $N_{0}$-базы остаются залитыми высококонцентрированной плазмой. На финальной стадии процесса обрыва тока плазма выносится из базовой области, и $p^{\prime} N_{0}$-переход запирается.
SOS-диоды являются мощными полупроводниковыми ключами размыкающего типа, работающими совместно с индуктивными накопителями энергии в высоковольтных генераторах импульсов наносекундной длительности. С практической точки зрения важными параметрами SOS-диодов являются: максимальное импульсное напряжение, плотность разрываемого тока, время переключения, а также коммутационные потери. Все эти параметры зависят от длительности, амплитуды и формы импульсов прямого и обратного тока, характеристик индуктивного накопителя и импеданса нагрузки. Однако в первую очередь эти параметры зависят от конструкции полупроводниковой структуры SOS-диода.

Детальный анализ процесса обрыва тока и дальнейшие исследования, выполненные в нашей лаборатории, выявили эффекты, непосредственно влияющие на эффективность SOS-диода как высоковольтного быстродействующего ключа.

Как уже было отмечено, в процессе обрыва тока SOSдиодом вблизи $n^{+} N_{0}$-границы и в $p^{\prime}$-слое возникают два плазменных фронта и два расширяющихся домена сильного поля, перемещающиеся навстречу друг другу. При этом домен, сформировавшийся в $p^{\prime}$-слое, движется значительно быстрее вследствие разницы подвижностей электронов и дырок в кремнии. В результате домены встречаются вблизи $N_{0} n^{+}$-границы, т.е. на расстоянии нескольких десятков $\mu \mathrm{m}$ от $p^{\prime} N_{0}$-перехода. К этому моменту пик напряжения на нагрузке уже сформирован, однако для завершения процесса обрыва тока и восстановления запирающих свойств диода необходимо удалить подвижные носители из области $p^{\prime} N_{0}$-перехода и восстановить квазинейтральность во всей базовой области. Требуемый для этого перенос носителей через области сильного поля приводит к дополнительным ком- 
мутационным потерям и затягиванию процесса обрыва тока. Оптимальной же с точки зрения восстановления является структура, в которой плазменные фронты встречались бы непосредственно на $p-n$-переходе SOS-диода.

Действительно к моменту достижения максимума напряжения на нагрузке большая часть базовой области и $p-n$-переход остаются залитыми плазмой. Последующий вынос этой плазмы через области сильного поля дает существенный вклад в общие потери. Электроны выносятся через область сильного поля у $n^{+} N_{0}$-границы, а дырки через область сильного поля в $p^{\prime}$-слое. Коммутационные потери пропорциональны произведению плотности тока проводимости на напряженность электрического поля:

$$
E_{\mathrm{loss}}=S \iint\left(J_{n}(x, t)+J_{p}(x, t)\right) E(x, t) d x d t,
$$

где $S$ - площадь $\operatorname{SOS}$-диода, $J_{n}(x, t)$ и $J_{p}(x, t)-$ плотности электронного и дырочного тока соответственно; $E(x, t)$ - распределение электрического поля в SOS-диоде.

Изменение профиля легирования SOS-диода и переход на кремний $p$-типа существенно улучшает ситуацию. Такая $p^{+} P_{0} n^{+}$-структура, названная $p$-SOS-диодом, была предложена в [5]. В пластину кремния $p$-типа проводимости с толщиной $\sim 210 \mu \mathrm{m}$ и удельным сопротивлением $\rho \approx 30-60 \Omega \cdot \mathrm{cm}$ с противоположных сторон проводится диффузия бора и фосфора с поверхностной концентрацией до $10^{20} \mathrm{~cm}^{-3}$ на глубину порядка $70 \mu \mathrm{m}$, a ширина базовой области $P_{0}$ составляет $60-80 \mu \mathrm{m}$. В работе [5] было экспериментально подтверждено наличие SOS-эффекта в диодах на базе кремния $p$-типа. $p$-SOS-диоды показали меньшее время переключения и существенно меньшие коммутационные потери по сравнению с SOS-диодами на базе $n$-кремния (n-SOSдиоды).

Численное моделирование процесса обрыва обратного тока высокой плотности в такой $p^{+} P_{0} n^{+}$-структуре, проведенное в [6], показало, что этот процесс, как и в $p^{+} p^{\prime} N_{0} n^{+}$-структуре SOS-диода, начинается с образования в $n^{+}$-слое у $P_{0} n^{+}$-перехода узкой области сильного поля. Этот пик поля быстро достигает величины $\sim 150 \mathrm{kV} / \mathrm{cm}$, после чего его форма и величина меняются медленно, т.е. правый плазменный фронт медленно перемещается к $P_{0} n^{+}$-переходу. В это время вблизи $p^{+} P_{0}$-границы формируется быстро расширяющаяся область сильного поля, приводящая к возрастанию сопротивления диодной структуры и перебросу тока в нагрузку. Левый плазменный фронт быстро пробегает по $P_{0}$-базе, удаляя из нее накопленную плазму. Фронты встречаются вблизи $P_{0} n^{+}$-перехода, после чего начинается быстрое восстановление диода. Таким образом, время, необходимое для полного обрыва тока в таком приборе, определяется в основном временем пробега левого плазменного фронта по $P_{0}$-базе, а также временем формирования области объемного заряда на
$P_{0} n^{+}$-переходе. Момент восстановления $p$-SOS-диода сопровождается резким падением остаточного тока через него, что наблюдалось как в эксперименте, так и в модели. Однако численное моделирование показало, что в момент формирования выходного импульса на нагрузке и максимума напряжения на $p$-SOS-диоде между плазменными фронтами остается область $P_{0}$-базы шириной порядка $30 \mu \mathrm{m}$, заполненная плазмой. Так же как и в $n$-SOS-диоде, последующий вынос этой плазмы через области высокого поля ведет к дополнительным коммутационным потерям. Кроме того, избыточная толщина базы увеличивает время пробега плазменного фронта по ней и замедляет обрыв тока SOS-диодом.

Поэтому следующим логичным шагом по пути оптимизации структуры $p$-SOS-диода явилось соответствующее уменьшение толщины $P_{0}$-базы. Экспериментальные результаты исследования такой структуры, а также численное моделирование динамики электронно-дырочной плазмы в ней представлены в настоящей работе.

\section{Описание модели}

Были исследованы оптимизированные $p$-SOS-диоды площадью $1 \mathrm{~mm}^{2}$, изготовленные из кремниевых пластин толщиной $210 \mu \mathrm{m} p$-типа проводимости с $\rho=60 \Omega \cdot \mathrm{cm}$; $p^{+}$-область была сформирована двумя последовательными диффузиями бора с поверхностными концентрациями и глубинами $2 \cdot 10^{18} \mathrm{~cm}^{-3}$ и $90 \mu \mathrm{m}, 8 \cdot 10^{19} \mathrm{~cm}^{-3}$ и $5 \mu \mathrm{m}$ соответственно, а $n^{+}$-область образована диффузией фосфора с поверхностной концентрацией $5 \cdot 10^{19} \mathrm{~cm}^{-3}$ и глубиной $90 \mu \mathrm{m}$. Таким образом, ширина $P_{0}$-базы составила $30 \mu \mathrm{m}$. Профиль легирования исследованной $p^{+} P_{0} n^{+}$-структуры и расчетная форма распределения электронно-дырочной плазмы в момент начала импульса

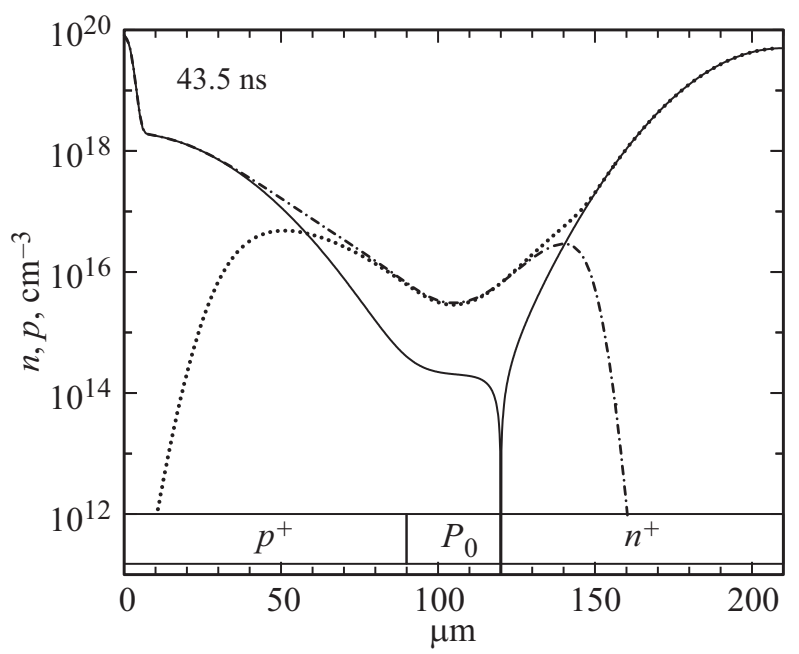

Рис. 1. Профиль легирования $p^{+} P_{0} n^{+}$-структуры SOS-диода $(-)$ и расчетная форма распределения электронов $(\ldots)$ и дырок $(\cdot-\cdot-\cdot)$ в плазме к моменту окончания импульса прямого тока. 


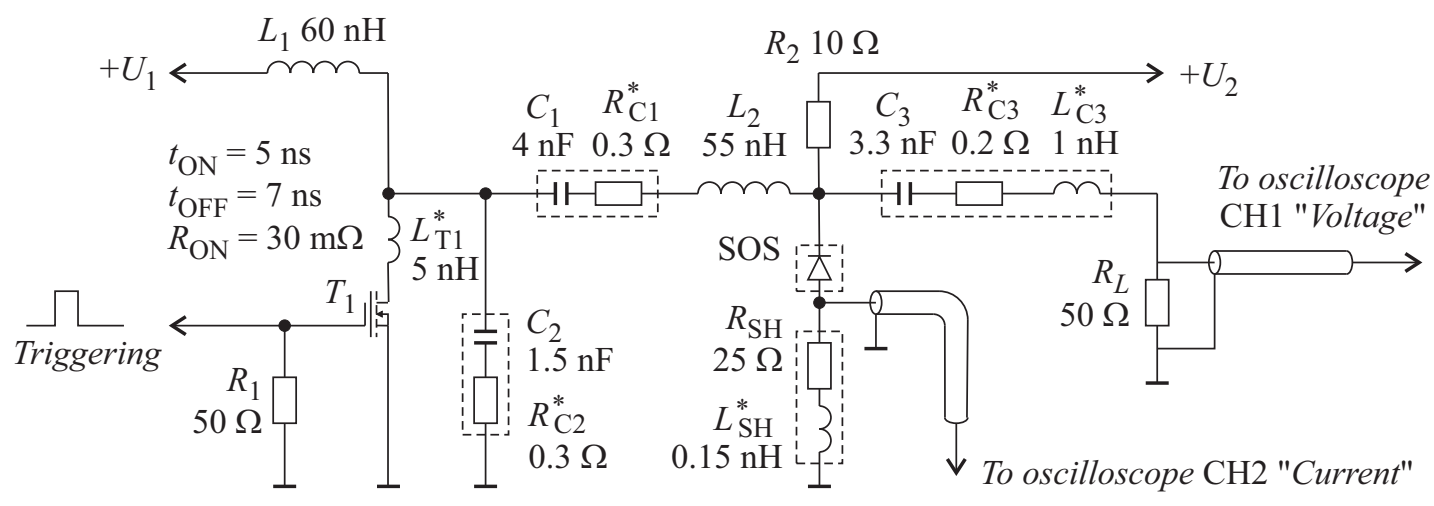

Рис. 2. Эквивалентная электрическая схема стенда для испытания SOS-диодов. Паразитные параметры соответствующих элементов обозначены *.

обратного тока после протекания импульса тока накачки показаны на рис. 1.

Моделирование выполнялось в программе Atlas пакета Silvaco путем совместного решения уравнений Кирхгофа для внешней эквивалентной электрической схемы и одномерной диффузионно-дрейфовой физической модели динамики электронно-дырочной плазмы в $p$-SOS-диоде. По сравнению с [6] был уточнен ряд параметров, входящих в схему компонентов, в том числе их паразитные параметры, учтена паразитная индуктивность шунта, измеряющего ток через $p$-SOS-диод, а также ограничение полосы пропускания измерительных трактов. Уточненная схема приведена на рис. 2, паразитные элементы отмечены знаком $(*)$. Тщательное измерение параметров всех электронных компонентов, включая их паразитные параметры, было необходимо для получения не только качественного, но и количественного согласия расчета с экспериментом.

Аналогичным образом необходимо было тщательно подойти к построению и выбору параметров диффузионно-дрейфовой физической модели $p$-SOS диода.

Для расчета процессов динамики электронно-дырочной плазмы в $p$-SOS-диоде использовалась фундаментальная система уравнений, состоящая из уравнений непрерывности, диффузионно-дрейфовых транспортных уравнений и уравнения Пуассона:

$$
\begin{gathered}
\frac{\partial n}{\partial t}=\frac{1}{e} \frac{\partial J_{n}}{\partial x}+G_{n}-R, \frac{\partial p}{\partial t}=-\frac{1}{e} \frac{\partial J_{p}}{\partial x}+G_{p}-R, \\
J_{n}=e D_{n} \frac{\partial n}{\partial x}+e n \mu_{n} E, J_{p}=-e D_{p} \frac{\partial p}{\partial x}+e p \mu_{p} E, \\
\frac{\partial E}{\partial x}=\frac{e}{\varepsilon}\left(p-n+N_{d}-N_{a}\right),
\end{gathered}
$$

где $n, p, N_{d}, N_{a}-$ концентрации свободных электронов, дырок, а также донорных и акцепторных примесей; $J_{n}, J_{p}-$ плотности электронного и дырочного тока; $G_{n}, G_{p}$ - скорости генерации электронов и дырок, рассчитываемые по модели Зельберхерра; $R$ - скорость рекомбинации, рассчитываемая по модели
Шокли-Рида-Холла; $D_{n}, D_{p}-$ коэффициенты диффузии электронов и дырок, которые в невырожденном полупроводнике в соответствии с соотношением Эйнштейна равны $\frac{k T_{L}}{e} \mu_{n}$ и $\frac{k T_{L}}{e} \mu_{p}$ соответственно; $T_{L}-$ температура кристаллической решетки; $\mu_{n}, \mu_{p}-$ подвижности электронов и дырок.

При расчете подвижности электронов и дырок учитывалась зависимость подвижности от электрического поля, рассеяние на колебаниях кристаллической решетки, электронно-дырочное рассеяние и рассеяние на ионизованных примесях. Для учета эффекта насыщения скорости носителей в сильных полях и соответственно уменьшения подвижности электронов и дырок была использована стандартная модель, предложенная в [7]. Эта модель обеспечивает плавный переход от слабых полей к сильным. Подвижность в слабых полях рассчитывалась по модели [8], которая является предпочтительной при высокой концентрации инжектированных носителей, характерной для силовых полупроводниковых приборов:

$$
\begin{gathered}
\mu_{n}=\mu_{n 0}\left[1+\left(\frac{\mu_{n 0} E}{v_{\mathrm{sat}}}\right)^{2}\right]^{-0.5}, \mu_{p}=\mu_{p 0}\left[1+\frac{\mu_{p 0} E}{v_{\mathrm{sat}}}\right]^{-1}, \\
v_{\mathrm{sat}}=\frac{2.4 \cdot 10^{7}}{1+0.8 \exp \left(\frac{T_{L}}{600}\right)}, \\
\mu_{n 0, p 0}=\mu_{n, p}^{L}\left[\frac{1.025}{1+\left(2.126 \frac{\mu_{n, p}^{L}}{\mu_{n, p}^{L}}\right)^{0.715}}-0.025\right] \\
\mu_{n}^{L}=1000\left(\frac{T_{L}}{300}\right)^{-1.5}, \mu_{p}^{L}=500\left(\frac{T_{L}}{300}\right)^{-1.5} \\
\mu^{C}=\frac{\mu_{n, p}^{I C}=\left(\frac{1}{\mu^{C}}+\frac{1}{\mu_{n, p}^{I}}\right)^{-1},}{\sqrt{n p} \ln \left[1+7.45 \cdot 10^{13}\left(\frac{T_{L}}{300}\right)^{2}(n p)^{-1 / 3}\right]} \\
\mu_{n}^{I}=\frac{4.61 \cdot 10^{17}\left(T_{L}\right)^{1.5}}{N_{T}} f\left(\frac{1.52 \cdot 10^{15}\left(T_{L}\right)^{2}}{n+p}\right)
\end{gathered}
$$




$$
\mu_{p}^{I}=\frac{1 \cdot 10^{17}\left(T_{L}\right)^{1.5}}{N_{T}} f\left(\frac{6.25 \cdot 10^{14}\left(T_{L}\right)^{2}}{n+p}\right),
$$

где $N_{T}=\Sigma_{i=1}^{n}\left|Z_{i}\right| N_{i}=N_{d}+N_{a}-$ полная концентрация ионизованных примесей; $f(x)=\left[\ln (1+x)-\frac{x}{1+x}\right]^{-1}$; вклад в подвижности электронов и дырок: $\mu_{n, p}^{I}-$ рассеяния на ионизованных примесях; $\mu^{C}-$ электроннодырочного рассеяния; $\mu_{n, p}^{L}-$ рассеяния электронов и дырок на кристаллической решетке; $\mu_{n 0, p 0}-$ подвижности электронов и дырок при слабых полях; $\mu_{n, p}-$ подвижности с учетом эффекта насыщения скорости носителей в сильных полях.

Для расчета рекомбинации электронов и дырок была взята упрощенная модель Шокли-Рида-Холла, применение которой допустимо при высоких уровнях инжекции, характерных для силовых полупроводниковых приборов. В модели также была учтена зависимость времени жизни носителей от концентрации примесей [9-11]. Согласно используемой модели, скорость рекомбинации равна

$$
\begin{gathered}
R=\frac{n p-n_{i}^{2}}{\tau_{p}\left[n+n_{i} \exp \left(\frac{E_{t}-E_{i}}{k T_{L}}\right)\right]+\tau_{n}\left[p+n_{i} \exp \left(\frac{E_{i}-E_{t}}{k T_{L}}\right)\right]}, \\
\tau_{n}=\frac{\tau_{n 0}}{1+\left(\frac{N_{T}}{N_{S R H N}}\right)}, \quad \tau_{p}=\frac{\tau_{p 0}}{1+\left(\frac{N_{T}}{N_{S R H P}}\right)},
\end{gathered}
$$

где $\tau_{n 0}=\tau_{p 0}=10 \mu \mathrm{s}-$ время жизни электронов и дырок в областях с низким уровнем легирования; $E_{t}-E_{i}=0$ - разница в энергиях между уровнем в запрещенной зоне, созданным примесью или дефектами, через который идет рекомбинация, и уровнем Ферми; коэффициенты $N_{S R H N}=N_{S R H P}=5 \cdot 10^{16} \mathrm{~cm}^{-3}$.

Для расчета процесса генерации носителей была использована локальная модель, предложенная Зельберхерром в [12], согласно которой скорости генерации носителей принимаются равными произведению коэффициентов ионизации $\alpha_{n}$ и $\alpha_{p}$ на плотности электронного и дырочного тока соответственно

$$
\begin{gathered}
G_{n}=\alpha_{n} J_{n}, \quad G_{p}=\alpha_{p} J_{p} \\
\alpha_{n}=A_{n} \exp \left[-\frac{B_{n}}{E}\right], \quad \alpha_{p}=A_{p} \exp \left[-\frac{B_{p}}{E}\right] .
\end{gathered}
$$

Температурная зависимость скорости генерации учтена в коэффициентах

$$
\begin{aligned}
& A_{n}=A_{n 1}\left(1+A_{n t}\left[\frac{T_{L}}{300}-1\right]\right), \\
& B_{p}=B_{p 1}\left(1+B_{p t}\left[\frac{T_{L}}{300}-1\right]\right),
\end{aligned}
$$

где $\quad A_{n 1}=7.03 \cdot 10^{5} \mathrm{~cm}^{-1}, \quad A_{n t}=0.588, \quad B_{n 1}=$ $1.231 \cdot 10^{6} \mathrm{~V} / \mathrm{cm}, \quad B_{n t}=0.248, \quad A_{p 1}=6.71 \cdot 10^{5} \mathrm{~cm}^{-1}$, $A_{p t}=0.588, B_{p 1}=1.693 \cdot 10^{6} \mathrm{~V} / \mathrm{cm}, B_{p t}=0.248$.

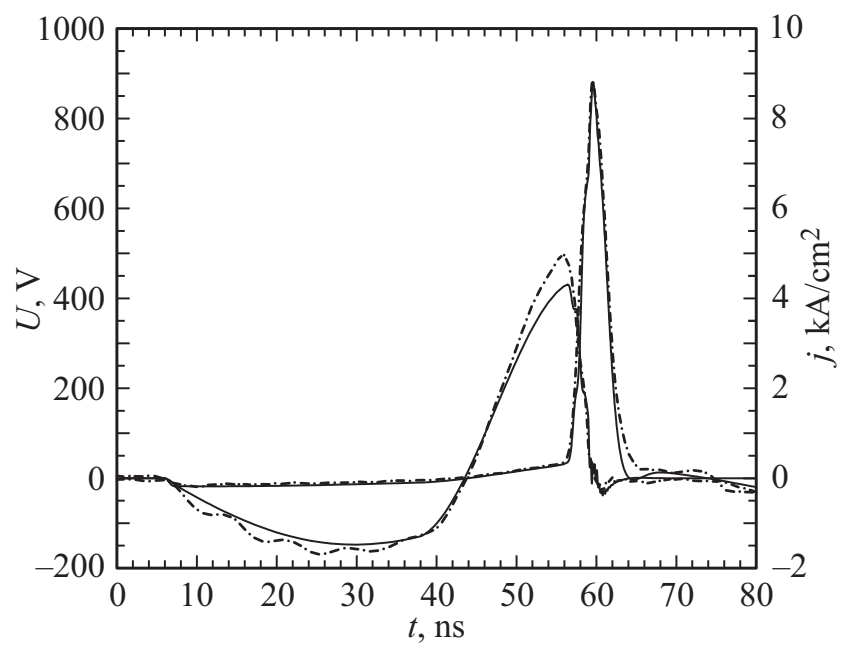

Рис. 3. Расчетные $(-)$ и экспериментальные $(\cdot-\cdot-\cdot)$ кривые прямого и обратного тока через $p$-SOS-диод, а также напряжения на параллельно подключенной нагрузке.

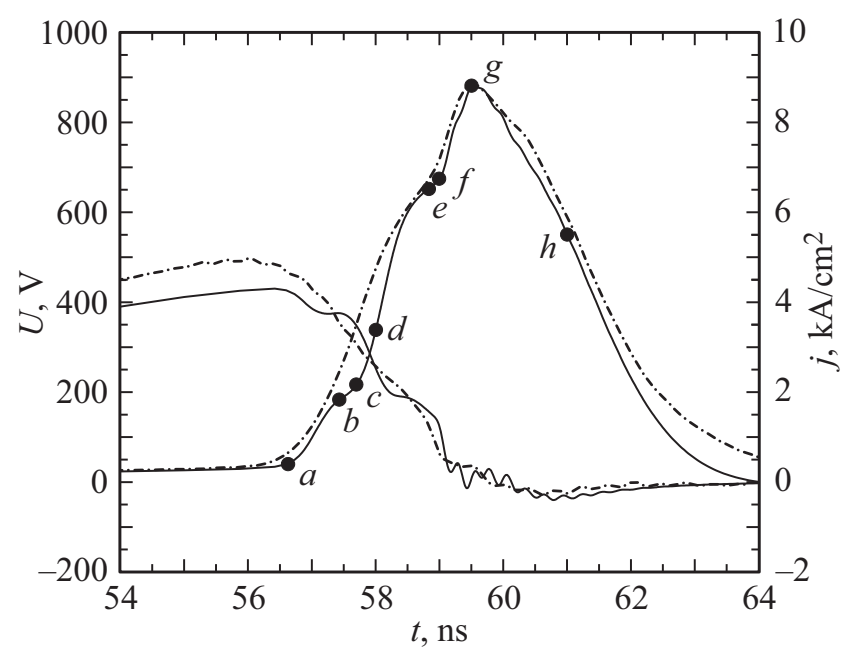

Рис. 4. Расчетные $(-)$ и экспериментальные $(\cdot-\cdot-\cdot)$ кривые обрыва тока $p$-SOS-диодом и формирования выходного импульса. Точками отмечены моменты времени, для которых приведен расчет распределения электронно-дырочной плазмы и электрического поля в структуре.

\section{Результаты моделирования и сравнение с экспериментом}

На рис. 3 показаны расчетные и экспериментальные формы импульсов прямого и обратно тока через исследуемую $p$-SOS-структуру, а также форма импульса напряжения на параллельно подключенной к диоду нагрузке $R=50 \Omega$. Более подробно процесс обрыва тока показан на рис. 4. Совпадение расчета и эксперимента достаточно хорошее. Буквами на кривых отмечены моменты времени, для которых приведены расчетные распределения концентрации носителей и напряженности электрического поля (см. ниже рис. 5). 

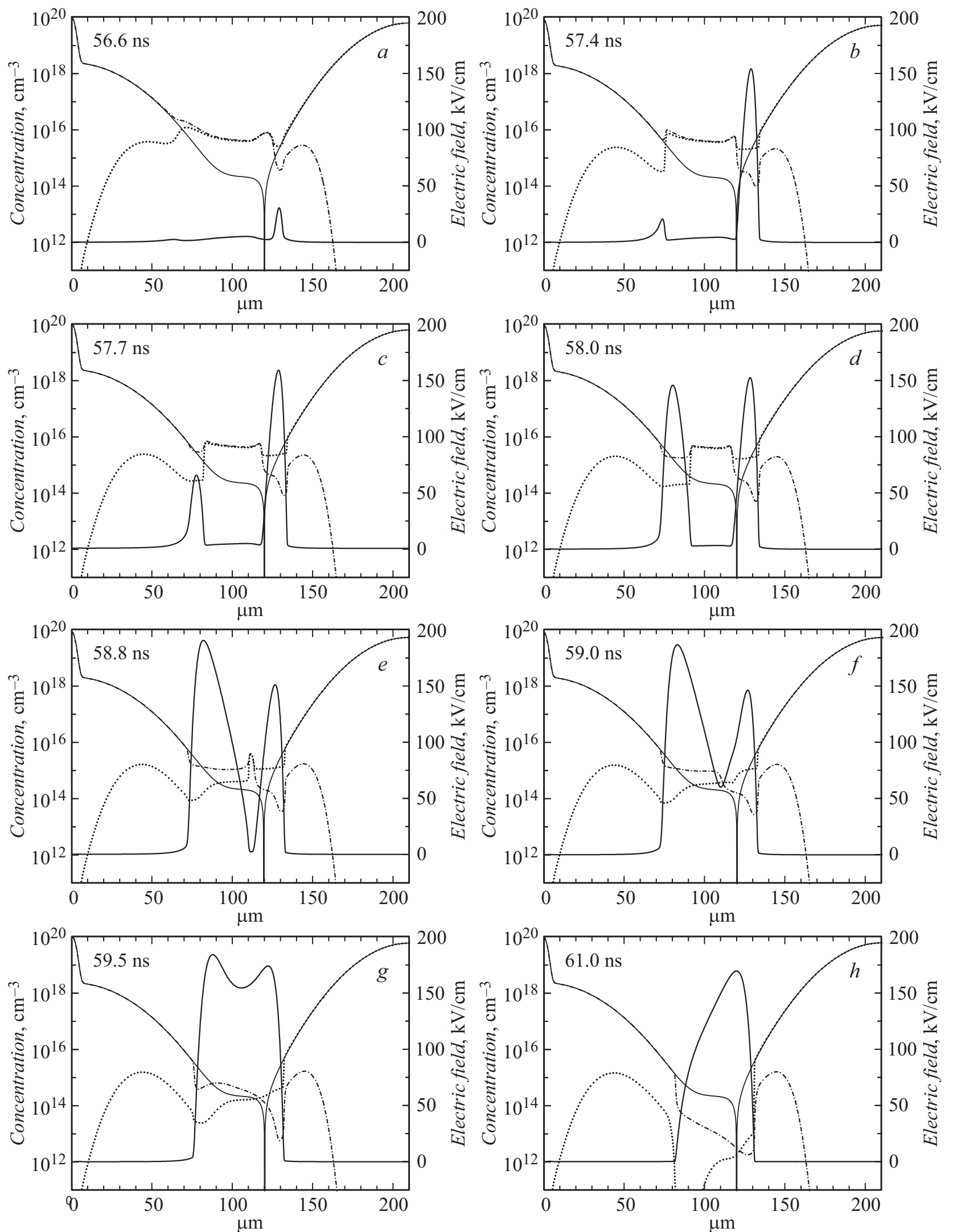

Рис. 5. Расчетные кривые распределения электронов $(\ldots)$ и дырок $(\cdot-\cdot-\cdot)$ в плазме, а также электрического поля $(-)$ в p-SOS-диоде в различные моменты времени в процессе обрыва тока. 
Как и $n$-SOS-диодах, процесс обрыва тока в $p$-SOS-диодах является многостадийным, что отражается в особенностях форм кривых тока и напряжения, наблюдаемых как экспериментально, так и при численном моделировании. Расчетные распределения электронно-дырочной плазмы и напряженности электрического поля в различные моменты времени показаны на рис. 5. На начальной стадии процесса обрыва тока базовая область залита высококонцентрированной плазмой, напряженность электрического поля в структуре невелика $(t=56.6 \mathrm{~ns}$; точка $a$ на рис. 4 и рис. $5, a)$. Аналогично с $n$-SOSдиодом первым возникает домен сильного поля в $n^{+}$области, который быстро достигает величины порядка $1.5 \cdot 10^{5} \mathrm{~V} / \mathrm{cm}(t=57.4 \mathrm{~ns}$, точка $b$ на рис. 4 и рис. $5, b)$, в то время как напряженность поля во втором домене в $p^{+}$-области все еще не превышает $2 \cdot 10^{4} \mathrm{~V} / \mathrm{cm}$, т.е. ниже примерно на порядок. На этой стадии в нагрузку переключается только небольшая часть силового тока, а напряжение на $p$-SOS-диоде достигает примерно 20\% от своей максимальной величины. Таким образом, на первой стадии переброс части силового тока в нагрузку происходит исключительно за счет возникновения и роста домена сильного поля в $n^{+}$-области. На второй стадии процесса обрыва тока амплитуда и положение домена сильного поля в $n^{+}$-области остаются практически неизменными, зато в $p^{+}$-области быстро расширяется и растет по амплитуде второй домен сильного поля $(t=57.7 \mathrm{~ns}$, точка $c$ на рис. 4 и рис. $5, c ; t=58.0 \mathrm{~ns}$, точка $d$ на рис. 4 и рис. $5, d)$. На этой стадии большая часть тока переходит в нагрузку, а напряжение выходного импульса достигает порядка 70\% от своего максимума. Продолжительность этой стадии определяется временем, необходимым для пробега плазменного фронта по $P_{0}$-базе, и именно эта стадия дает наибольший вклад в длительность фронта выходного импульса. В момент $t=58.8 \mathrm{~ns}$ (точка $e$ на рис. 4 и $5, e$ ) плазменные фронты смыкаются вблизи $P_{0} n^{+}$-перехода, и начинается третья стадия процесса обрыва тока, на которой домены сильного поля, имеющие форму, близкую к треугольной, перестраиваются в один общий домен сильного поля, имеющий квазипрямоугольный профиль распределения поля $(t=59.0 \mathrm{~ns}$, точка $f$ на рис. 4 и $5, f ; t=59.5 \mathrm{ns,}$ точка $g$ на рис. 4 и $5, g)$. Квазипрямоугольный профиль поля позволяет получить дополнительный выигрыш в амплитуде выходного импульса и напряжение на $p$ SOS-структуре, значительно превышающее напряжение статического пробоя $P_{0} n^{+}$-перехода. На этой стадии бо́льшая часть плазмы оказывается вынесенной из базовой области и области $P_{0} n^{+}$-перехода, ток через диод резко падает и полностью переходит в нагрузку. Напряжение на $p$-SOS-диоде достигает максимальной величины $900 \mathrm{~V}$. На этом формирование выходного импульса завершается, напряжение на диоде падает, а на $P_{0} n^{+}$-переходе формируется область объемного заряда с квазитреугольным распределением электрического поля, характерным для обратносмещенного диода $(t=61.0 \mathrm{~ns}$, точка $h$ на рис. 4 и $5, h)$.
Следует отметить, что для всех исследованных SOS-структур образование узкой области сильного поля в $n$-слое всегда начинается раныше образования широкой основной области сильного поля в $p$-слое. Это несколько затягивает процесс обрыва тока и увеличивает коммутационные потери.

Параметры процесса выключения (пиковое напряжение и время обрыва тока), приведенные в настоящей работе, скорее всего, близки к предельным для исследованных $p^{+} P_{0} n^{+}$-структур, поскольку пиковое значение напряженности поля в ходе процесса достигает величины $\sim 2 \cdot 10^{5} \mathrm{~V} / \mathrm{cm}$, выше которой начинается лавинный пробой. Эксперименты показывают, что разрушения структуры при этом не происходит, но интенсивная генерация электронно-дырочной плазмы приводит к затягиванию процесса обрыва тока, а также ограничению амплитуды и расширению выходного импульса напряжения на нагрузке. Для приведенной на рис. 3 предельной осциллограммы процесса выключения $p^{+} P_{0} n^{+}$ структуры пиковая плотность тока составляет $5 \mathrm{kA} / \mathrm{cm}^{2}$, время нарастания тока фронта выходного импульса около $2.3 \mathrm{~ns}$, а пиковое напряжение на нагрузке $900 \mathrm{~V}$, т. е. пиковая коммутируемая мощность $4.5 \cdot 10^{6} \mathrm{~W} / \mathrm{cm}^{2}$.

\section{Заключение}

В настоящей работе исследована $p^{+} P_{0} n^{+}$-структура SOS-диода с $P$-базой, уменьшенной до оптимальной толщины. Исследование проводилось как экспериментально, так и методом численного моделирования. Построена одномерная диффузионно-дрейфовая модель динамики электронно-дырочной плазмы, дающая хорошее согласие с экспериментом. Уменьшение толщины $P$-базы по сравнению со структурой, описанной в [6], позволило повысить амплитуду выходного импульса в два раза при той же плотности коммутируемого тока. Это было достигнуто за счет существенного снижения коммутационных потерь, а также за счет формирования в процессе обрыва тока области сильного электрического поля квазипрямоугольной формы на $P_{0} n^{+}$-переходе. В результате были сформированы выходные импульсы напряжения с амплитудой, существенно превышающей напряжение статического пробоя $P_{0} n^{+}$-перехода $p$-SOS диода. Этот эффект наблюдается впервые для высоковольтных полупроводниковых ключей размыкающего типа и требует дополнительного детального исследования.

Исследование выполнено за счет гранта Российского научного фонда (проект № 14-29-00094).

\section{Список литературы}

[1] Котов Ю.А., Месяи Г.А., Рукин С.Н., Филатов А.А. // ДАН. 1993. Т. 330. Вып. 3. С. 315-317.

[2] Рукин С.Н., Цыранов С.Н. // ФТП. 2009. Т. 43. Вып. 7. C. 989-995. 
[3] Любутин С.К., Рукин С.Н., Словиковский Б.Г., Цыранов С.Н. // ФТП. 2012. Т. 46. Вып. 4. С. 535-543.

[4] Engelko A., Bluhm H. // J. Appl. Phys. 2004. Vol. 95. N 10. P. 5828-5836.

[5] Грехов И.В., Люблинский А.Г., Белякова Е.И. // ЖТФ. 2016. Т. 86. Вып. 3. С. 106-109.

[6] Подольская Н.И., Люблинский А.Г., Грехов И.В. // ЖТФ. 2017. Т. 87. Вып. 12. С. 1790-1793.

[7] Caughey D.M., Thomas R.E. // Proc. IEEE. 1967. Vol. 55. P. 2192-2193.

[8] Dorkel, J., Leturcq Ph. // Solid State Electron. 1981. Vol. 24. P. 821-825.

[9] Roulston D.J., Arora N.D., Chamberlain S.G. // IEEE Tr. Electron. Dev. 1982. Vol. 29. P. 284-291.

[10] Law M.E. et al. // IEEE Electron. Dev. Lett. 1991. Vol. 12. N 8. P. 401-403.

[11] Fossum J.G., Lee D.S. // Solid State Electron. 1982. Vol. 25. P. 741-747.

[[12] Selberherr S. // Analysis and Simulation of Semiconductor Devices. Wien, NY.: Springer-Verlag, 1984. 296 p. 